\title{
MÁS ALLÁ DE LAS OBSERVACIONES DE CLASES DE INGLÉS EN COLOMBIA
}

Martínez-Luengas, Miguel

MÁS ALLÁ DE LAS OBSERVACIONES DE CLASES DE INGLÉS EN COLOMBIA

PANORAMA, vol. 15, núm. 28, 2021

Politécnico Grancolombiano, Colombia

Disponible en: http://www.redalyc.org/articulo.oa?id=343965146004

Institución Universitaria Politécnico Grancolombiano

Institución Universitaria Politécnico Grancolombiano

\section{(ㄷ) $(1) \Theta(9$}

Esta obra está bajo una Licencia Creative Commons Atribución-NoComercial-SinDerivar 4.0 Internacional. 
Artículos de investigación científica y tecnológica

\title{
MÁS ALLÁ DE LAS \\ OBSERVACIONES DE CLASES DE \\ INGLÉS EN COLOMBIA
}

\author{
Miguel Martínez-Luengas \\ miguel.martinez.gestor@gmail.com \\ Pontificia Universidad Javeriana, Colombia \\ (D) https://orcid.org/0000-0002-0456-2604
}

PANORAMA, vol. 15, núm. 28, 2021

Politécnico Grancolombiano, Colombia

Recepción: 06 Agosto 2020

Aprobación: 11 Noviembre 2020

Redalyc: http://www.redalyc.org/ articulo.oa?id=343965146004
Resumen: El presente escrito es una reflexión basada en las prácticas de observación en clases de inglés en Colombia. Es necesario recalcar que las observaciones de clases no solo son prácticas para evaluar, criticar o juzgar; a su vez, estas prácticas se constituyen como escenarios propicios en los cuales la reflexión y la crítica cobran un papel preponderante. Igualmente, se realizó un análisis de una estrategia específica, como lo son las observaciones de clases de inglés en Colombia, desde el año 2012 hasta 2019, en fuentes como programas de bilingüismo, planes de fortalecimiento de inglés y noticias de periódicos colombianos. El autor indaga sobre lo oculto en estas prácticas, lo que no se ve en las investigaciones académicas sobre el tema, y lo que probablemente no se debe mostrar cuando el observador y el observado se encuentran en esta dinámica de observación. Los hallazgos muestran una postura diferente y crítica sobre la estrategia de las observaciones de clases de inglés de Colombia por medio de unas categorías propuestas y, finalmente, el lector logrará identificar lo que posiblemente está más allá de estas prácticas que aún se llevan a cabo durante procesos educativos en este país.

Palabras clave: Desarrollo profesional docente, observación de clases, procesos de reflexión, sujeto observador.

Abstract: This present text is a reflection based on the classroom observation practices of English classes in Colombia. There is a need to highlight that classroom observations are not just practices evaluating, criticize or judge: at the same time, these practices are important processes where the reflection and the critical aspects play a relevant role. Also, an analysis from 2012 until 2019 of a strategy called classroom observations of English classes in Colombia was made in data sources such as Bilingualism programs, Strengthening English plans, and news on Colombian journals. The author inquiries into the hidden aspects of this practice, the unseen of this topic in research and probably something which should not be shown when the observer and the observed are interacting in this observation dynamic. The findings expose a different/critical stand about the classroom observations of English classes in Colombia through some categories proposed and then, the reader will be able to identify some aspects which are probably beyond in this practice and has still carried out during education processes in this country.

Keywords: Teachers' professional development, classroom observation, reflective processes, observer subject.

Resumo: O presente texto é uma reflexão baseada nas práticas de observação das aulas de inglês na Colômbia. É necessário destacar que as observações em sala de aula não são apenas práticas para avaliar, criticar ou julgar, más são processos importantes nos quais a reflexão e os aspectos críticos desempenham um papel relevante. Uma análise das observações das aulas de inglês de 2012 a 2019, na Colômbia, foi realizada em fontes de dados, como programas de bilinguismo, planos do governo para o fortalecimento da Língua inglesa e notícias em jornais colombianos. $\mathrm{O}$ autor investiga os aspectos ocultos dessa prática, o invisível deste tópico na pesquisa e, provavelmente, 
algo que não deve mostrar quando o observador e o observado estão interagindo nessa dinâmica de observação.As descobertas apresentam uma posição diferente/crítica sobre as observações das aulas de inglês na Colômbia através de algumas categorias propostas e, finalmente, o leitor poderá identificar o que está além dessa prática e o que foi realizado durante os processos educacionais neste país.

Palavras-chave: Desenvolvimento profissional dos professores, observação em sala de aula, processos reflexivos, sujeito observador.

\section{INTRODUCCIÓN}

Las observaciones de clases de inglés son evidenciadas en los procesos de formación docente y evaluación de desempeño, así como para brindar información sobre los estándares de calidad en programas de gobierno del país y proporcionar datos en proyectos de educación, entre otros.

Si bien la observación de clase en el área de inglés en Colombia es utilizada como una técnica de recolección de información, y de gran ayuda para llevar a cabo investigaciones académicas y científicas, también permite reflexión sobre las prácticas docentes diarias; con base en esto es importante explorar sobre nuestra propia dinámica docente, que también se materializa por medio de la observación (Lasagabaster, 2006).

No obstante, surgen algunas preguntas: ¿cuál es la importancia de observar al profesorado de inglés?, ¿cuáles son los elementos específicos que debe tener en cuenta el observador a la hora de realizar su trabajo?, ¿se siente bien el profesorado de inglés al ser observado?, ¿cuáles sensaciones son consideradas por el observador/observado a la hora de esta práctica? El fin principal no es dar soluciones a estas interrogantes, sino entender la posición del observador, el observado y lo encubierto en las prácticas de observación durante la lectura de este texto. Al mismo tiempo, se deja al lector la invitación a reflexionar sobre su propia experiencia en este proceso y a indagar sobre posibles "opciones" al momento de vivir una práctica de observación de clases.

El material utilizado hace referencia a un estudio (elaborado por el autor de este artículo, del año 2002 hasta 2019), mostrado a manera de análisis, donde el lector percibe las dinámicas que ocurrieron durante los procesos de observación de clases de inglés en Colombia, entre ellas las estrategias del gobierno nacional, el rendimiento profesional docente y la capacitación pedagógica del profesorado de inglés.

Más aún, los resultados del presente artículo buscan abrir el panorama sobre las OCIC, que no deben ser consideradas solo como prácticas de juicio o como medio de evaluación y desarrollo profesional docente. Se muestran algunas categorías que emergieron en este estudio. También se argumentan lo que no muestran los textos sobre las observaciones, lo que las cifras o los datos estadísticos no revelan en las prácticas de OCIC; tal vez sea posible llegar a ofrecer una mirada distinta sobre los encuentros entre el observador y el docente observado, lo invisible... lo que no se ve en estas conversaciones entre estos dos sujetos y lo significativo durante el ejercicio de observar al otro.

En esta reflexión, el lector encontrará particularidades biográficas sobre la observación de clase, situaciones por las que han pasado los 
investigadores, observadores de clases y profesores observados. También se presentarán apartados sobre autores especializados en este tema; se busca dar profundidad a las preguntas iniciales y cuestionar crítica y reflexivamente las prácticas de observaciones de clases de inglés en Colombia a lo largo de los últimos años. En último lugar, el artículo presenta una conclusión a manera de reflexión, con posturas críticas y analíticas derivado de las observaciones de clases, los procesos que se llevan a cabo allí y las rúbricas utilizadas en las observaciones de clase de inglés en Colombia.

\section{METODOLOGÍA}

Este artículo de reflexión considera un enfoque cualitativo de investigación, el cual se basa en el análisis de las observaciones de clases de inglés en Colombia. Se indagó sobre un archivo en donde planes de gobierno, estrategias de fortalecimiento del idioma inglés y algunos periódicos refieren las observaciones de clases de inglés como situaciones para promover el desarrollo profesional docente, evaluar los parámetros vinculados al aula de clase y dar seguimiento a los estándares regulados por políticas educativas de gobierno; esta investigación, a su vez, se constituye como una investigación interpretativa, expuesta a lo particular.

Además, la construcción de la teoría es evidenciada a partir de efectuar continuas reflexiones durante la inmersión profunda en el campo sobre los datos recolectados y sus impresiones respecto al contexto específico (Hernández, Fernández, \& Baptista, 2008).

En lo que se refiere a la metodología, el texto presenta la tabla 1 como columna vertebral, lo que permitirá construir unos resultados a manera de categorías para su análisis y reflexión posterior. El análisis en el material se realizó como un ejercicio de producción intelectual durante los estudios doctorales del autor de este artículo, en un seminario denominado Critical Discourse Analysis (en español, Análisis Crítico del Discurso), sobre las prácticas de observaciones de clases de inglés, que es la temática estudiada durante el proyecto de investigación. Se indagó sobre las diferentes características que tienen las prácticas de observaciones de clases en Colombia desde 2012 hasta 2019 en programas/planes de gobierno nacional, estrategias de fortalecimiento de inglés de instituciones externas y archivos de algunos periódicos colombianos en los que el tema surgió por medio de noticias y relatos. La recolección de datos se realizó con base en el contexto local colombiano, y no se manejó un gran volumen de información, lo cual permitió una técnica de análisis de contenido simple para identificar la información beneficiosa en este estudio (Tinto, 2013).

La tabla 1 está dividida en dos partes. La primera hace referencia a las fuentes de información en las que cuales emergieron como tema observaciones de clases de inglés, seguido de información tomada de la fuente original y aquella en la que el autor identificó apartados relacionados con el tema en cuestión. La invitación es a considerar las 
diversas particularidades que abarca el tema de observaciones de clase de inglés en Colombia.

\begin{tabular}{|c|c|}
\hline Fuento & Descripción \\
\hline $\begin{array}{l}\text { 1. Plan de bilingüismo } \\
\text { Municipal Mosquera } \\
\text { (2012). } \\
\text { Mosquera Lives English } \\
\text { 2012-2021 }\end{array}$ & $\begin{array}{l}\text { Según el Plan de bilingüismo, fa observación de clases se definió como una herramienta valiosa para el } \\
\text { mejoramiento del desempeño docente, porque esta práctica identifica los componentes y brinda retroalimentación } \\
\text { formativa al desempeño docente en el aula (p. } 15 \text { ). } \\
\text { Se organizan sesiones de talleres que estarian acompañados de observación de clase constante en el aula con } \\
\text { cada uno de los docentes, de manera que reciban retroalimentación formativa sobre uso práctico de los temas vistos } \\
\text { en los talleres (p.47). }\end{array}$ \\
\hline $\begin{array}{l}\text { 2. MEN (2014), Colombia } \\
\text { Very Well - Programa } \\
\text { nacional de inglés 2015- } \\
2025\end{array}$ & $\begin{array}{l}\text { Una de las estrategias del programa se basa en la creación de comunidades docentes, las cuales permiten } \\
\text { compartir experiencias de enseñanza, aprender mutuamente y desarrollar y diseminar mejores prácticas (por } \\
\text { ejemplo: observación de pares, estudio de lecciones, clases abiertas, etc.). } \\
\text { Uno de los objetivos de la observación de pares es la observación mutua, para que los docentes identifiquen } \\
\text { oportunidades de mejora en la práctica docente (p.74). }\end{array}$ \\
\hline $\begin{array}{l}\text { 3. Hernández Santamaría } \\
\text { (2016), } \\
\text { ¡Bienvenidos los cambios! } \\
\text { Evaluación integral a los } \\
\text { docentes de nuestro pais. }\end{array}$ & $\begin{array}{l}\text { Como parte clave de esta referencia, la observación de pares permite ver, al menos en parte (pues no es un video } \\
\text { sorpresa o cámara escondida). } \\
\text { T...] El video deberà ser grabado por el profesor, ya sea con recursos propios o con camarógrafo del ICFES, sin costo } \\
\text { adicional. Se debe garantizar la calidad de la auto grabación, por lo cual el MEN ha dispuesto manuales y videos } \\
\text { informativos, tanto para la grabación como para adecuar la prática docente a evaluar [.... }\end{array}$ \\
\hline $\begin{array}{l}\text { 4. MEN (2016), Colombia } \\
\text { bilingüe. } \\
\text { Programa de formadores } \\
\text { nativos extranjeros }\end{array}$ & $\begin{array}{l}\text { Según esta estrategia, los formadores nativos extranjeros (fellows), "apoyan a los gestores de bilingüismo en el } \\
\text { proceso de observación de clases en sus visitas a la IE" (p.67). }\end{array}$ \\
\hline $\begin{array}{l}\text { 5. British Council- } \\
\text { Secretaria de Educación } \\
\text { Distrital. } \\
\text { Convenio } 1550 \text { de } \\
2017\end{array}$ & $\begin{array}{l}\text { Como parte del proceso de las Escuelas formadoras y Maestros de maestros, se formuló una "Guia de rondas de } \\
\text { observación de clase". } \\
\text { Algunos objetivos de la ronda de observación son: } \\
\text { 1. Aproximar a los Maestros en formación a los Referentes de progreso. } \\
\text { II. Reconocer que los Referentes de progreso son los elementos constitutivos de una buena práctica docente, y } \\
\text { alrededor de los cuales se hará la retroalimentación de la práctica. } \\
\text { III. Iniciar una cultura de observación de clase en los Maestros en formación (MF), como medio para una buena } \\
\text { práctica de aula" (p.2). }\end{array}$ \\
\hline $\begin{array}{l}\text { 6. MEN (2018-2019), } \\
\text { Manual para } \\
\text { autograbación del video } \\
\text { de práctica. } \\
\text { Evaluación de carácter } \\
\text { diagnóstico formativa } \\
\text { (ECDF) }\end{array}$ & $\begin{array}{l}\text { En la evaluación de carácter diagnóstico formativa (ECDF), se evidencia un manual para consulta en general en el } \\
\text { que se garantiza la calidad de la autograbación (autoobservación) de la práctica educativa y pedagógica como } \\
\text { docente de aula. } \\
\text { Otra situación tiene que ver con que el video de la práctica educativa debe ser parte del periodo en el que el } \\
\text { profesor está siendo evaluado y cumplir con las disposiciones contempladas en la normatividad ( } p .5 \text { ). }\end{array}$ \\
\hline $\begin{array}{l}\text { 7. Cabrales ( } 7 \text { de junio de } \\
\text { 2019), Las } 2 \text { Orillas } \\
\text { "Las fallas del examen de } \\
\text { ascenso docente". }\end{array}$ & $\begin{array}{l}\text { †...] En la ECDF se evalúa a varios profesores colombianos para determinar si son ascendidos o reubicados } \\
\text { salarialmente. Una de las técnicas consiste en grabar un video. Con base en el escritor, argumenta lo siguiente: 'El } \\
\text { video no es más que una farsa, un espectáculo banal cuyo fin - pareciera- es propiciar una renta al Icfes y a } \\
\text { ciertas universidades que participan en ese proceso y recaudan cierta cuantia monetaria importante". }\end{array}$ \\
\hline
\end{tabular}

\section{Tabla 1.}

Observaciones de clases de inglés en Colombia 2012-2019

Fuente: elaboración propia, durante el seminario Critical Discourse Analysis, Doctorado Interinstitucional en Educación de la Universidad Distrital FJDC, Bogotá, 2019.

Conviene subrayar que la tabla 1 tiene tan solo siete fuentes primarias de información, y el análisis es planteado del año 2012 al año 2019. Entre los referentes teóricos de observaciones de clases de inglés hay varios autores, académicos e investigadores que han escrito y hablado sobre este tema; sin embargo, la idea al escribir este artículo radica en la posibilidad de hacer un análisis sobre algunos de los programas de gobierno de educación nacional, planes de bilingüismo, estrategias de mejoramiento de calidad educativa de entidades externas y noticias en periódicos locales en los que emergieron observaciones de clases. Además, se identifican diversos puntos de vista sobre lo que está más allá en las observaciones de clases de inglés en Colombia. A continuación, se encuentran las categorías que se manifestaron al explorar este análisis. Se pretende sustentar lo que se ha estudiado y lo que aún no se ha dicho sobre las observaciones de clases de inglés en Colombia. 


\section{RESULTADOS}

En cuanto a los resultados propuestos en este texto, es importante resaltar que la información obtenida en la tabla 1 abarca tan solo unos fragmentos en los que las prácticas de observaciones de clases eran evidentes (la información de cada una de las fuentes es amplia en contenido); así mismo, se proponen unas categorías emergentes en dicho análisis para intentar cubrir las siete fuentes descritas. A continuación, se especificarán las categorías que sobresalen como resultados de esta investigación.

\section{La evaluación y realimentación en las observaciones de clases de inglés}

Antes de delimitar esta categoría -basándose en las observaciones de clases de inglés- es preciso referir que el Diccionario de la Real Academia Española define el verbo evaluar como: "atribuir un valor a algo o a alguien, en función de un proyecto implícito o explícito", y, a su vez, la retroalimentación es la "Acción y efecto de realimentar". Partiendo de esto, las observaciones de clases de inglés son procesos en los cuales se atribuye algo particular, y a la vez hay una valoración; es decir, se da una nota/valor, pero se reciben comentarios del porqué de esa nota.

En clases de inglés es común evaluar los procesos pedagógicos y académicos por medio de la observación de clases. Si bien, al estar en colegios e institutos de inglés en las que las clases son observadas con el fin de evaluar los procesos, también se evalúa una(s) metodología(s) propuestas en un syllabus (lineamientos de clases), y si se está haciendo "bien" la labor como profesor de inglés. Partiendo de la vivencia particular como profesor de inglés, surgen muchas veces las preguntas: ¿qué es hacer una "buena" labor?, ¿es una buena labor el seguimiento de actividades planeadas y resueltas al pie de la letra? Por ahora, indagar acerca de la buena o mala práctica del profesor dentro y fuera del aula no viene al caso, ¡o tal vez sí! Lo interesante de esta primera categoría sobre la evaluación y retroalimentación en las observaciones de clases es profundizar un poco más en lo que está descrito o lo que usualmente se muestra en los libros de capacitación docente, y a lo mejor lo que no se ve o se encuentra oculto.

Como lo nombran Martínez, Cruz, Bamond, Fernández y Strotmann (2018), es importante tener en cuenta el detalle de los métodos y las técnicas de observación. También argumentan, según Gosling (2002), lo siguiente:

El modelo de desarrollo (development model) [es] donde un profesor experto o con más años de experiencia realiza una evaluación sumativa o de diagnóstico a otro profesor. En este modelo se busca el beneficio del observado. Y el modelo de revisión entre pares (peer review) [es] donde la relación entre observado y observador es de igualdad, y se busca el beneficio de ambos. (p.3)

No es un secreto que, en el contexto colombiano, el profesor "experto", es decir, con más años de experiencia, más formación académica, con un puesto/cargo en un nivel más alto, entre otros aspectos, es el indicado para observar una clase de inglés a un profesor que está iniciando su experiencia 
laboral o no tiene los atributos del otro. Los dos sujetos recibirán a cambio algún tipo de incentivo en su desarrollo profesional: el observador crece profesionalmente al suministrar conceptos de análisis, evaluación y crítica, mientras que el observado se enriquece de los comentarios y aportes significativos que se brinden.

Lo descrito antes es llamado peer observation (observación de pares), durante la cual colegas o compañeros de trabajo se observan el uno al otro para beneficiarse mutuamente en sus prácticas diarias, además de hacer un ejercicio de realimentación. Podemos notar esto en la tabla 1 (fuentes 1 y 2), cuando exponen "retroalimentación formativa sobre uso práctico de los temas vistos en los talleres", y también "el objetivo es que a partir de la observación mutua se identifiquen oportunidades de mejora en la práctica docente", respectivamente.

En este sentido, algo oculto en las observaciones de clase de inglés (evaluación y realimentación), tiene que ver con que no se especifican las personas "expertas" que observan las clases y brindan una realimentación. Además, en casi todos los procesos educativos se tiende a evaluar mediante una observación lo que se ve en directo/de inmediato, lo que el ojo experto puede concluir al ver las prácticas del profesor. Un coordinador, algún directivo o un observador externo observa una clase de inglés, y si el profesor no cumplió de manera satisfactoria su objetivo en esa precisa clase, el resultado es una mala calificación, que incluso puede poner en riesgo su puesto.

Hay otros métodos de evaluar (en opinión del autor de este documento), que podrían ser importantes. Uno de estos sería preguntar a los estudiantes antes y después de observar. Los estudiantes muchas veces generan esa confianza al evaluar al profesor; ellos están directamente implicados en los procesos académicos, y lograr tener una charla con los estudiantes puede generar más datos que una observación de clase. Es decir, la observación no debe volverse el dispositivo primordial para evaluar y juzgar la labor del profesor dentro y fuera del aula de clases. El diálogo entre profesor y observador, las narrativas de los mismos profesores o estudiantes en cuanto al desarrollo de la clase, o una charla informal con la comunidad académica pueden ser consideradas alternativas para indagar en las dinámicas de clases del profesorado.

No obstante, ¿quién nombra a las personas expertas para observar las clases?, ¿cómo obtuvieron ese título?, ¿por qué debe ser una relación vertical entre el observador y el observado?, ¿las observaciones de clases son un posicionamiento jerárquico?

Estas son preguntas que se derivan de este análisis. Se ubican en el más allá de las observaciones, y es relevante que el profesorado de inglés analice dichas situaciones, genere sus propias reflexiones y logre tener un plan de acción al enfrentarse a procesos de evaluación y realimentación. Estos dos últimos procesos no son los únicos identificados en las observaciones de clases; existen otros que se verán en los siguientes apartados. 


\section{El control, la critica y la disciplina}

Antes de justificar de lleno el tema, es conveniente citar lo que dicen Rekalde, Vizcarra y Macazaga (2014): "La observación es un método interactivo de recogida de información que requiere de la implicación del observador en los acontecimientos observados, ya que permite obtener percepciones de la realidad estudiada [...]" (citados por Rodríguez, Gil, \& García, 1996, p.7).

Aunque se tiene en cuenta esto, la observación no se trata tan solo de recolectar información; es un desarrollo en el que se involucran varios acontecimientos, tanto en contextos escolares, como en desarrollo profesional docente y procesos de calidad educativa. El control, la crítica y la disciplina son particularidades en la observación de clases, y en este caso específico, en clases de inglés.

Desde la experiencia obtenida como profesor de inglés y observador de clases del autor del presente texto, es posible decir que hay un control al querer impartir la praxis por medio de los lineamientos establecidos desde hace décadas y validados tanto por el profesor como por demás académicos del campo y la sociedad en general. Estos lineamientos, por supuesto, están acompañados de una disposición del observador, quien mediante una rúbrica de evaluación controla el paso a paso de las dinámicas del profesor en el aula. Hay un control en las clases de inglés, ya que la observación se centra en ver un insistente paso a paso de actividades, un sistema de evaluación universal-moderno y una estructura rígida en el manejo de clases según las necesidades del contexto. A mi modo de ver, las clases de inglés están dispuestas a perdurar en un sistema homogéneo, y evidentemente las observaciones de clases se centran en esto, en algo que se encuentra ratificado por el hombre, una práctica normalizada en procesos educativos actuales. ¿Qué control hay mediante una observación de clase?

Los directivos de instituciones educativas, observadores pares, entidades de gobierno (como ministerios y secretarías locales), necesitan obtener datos sobre las prácticas del profesorado dentro del aula. ¿Cómo se logra esto? Una observación de clases brindará estos números/cifras para identificar las falencias, mejorar a partir de los errores y multiplicar las "buenas" prácticas.

Esto se asemeja mucho a un sistema de control y disciplina en el sistema que debe ser propuesto en cada una de las acciones al observar una clase. Si se revisa el análisis en la tabla 1 , fuente 3, se puede detallar que, según el MEN "Evaluación de Carácter Diagnóstico Formativa" (ECDF), y en palabras de Hernández, "la Evaluación de Carácter Diagnóstico Formativa se trata de una metodología que ofrece mayor objetividad e integralidad en el diagnóstico del ejercicio docente, pues se enfoca en la práctica pedagógica, atiende al contexto particular de cada docente". Esta nueva evaluación docente trata sobre un control, ya que, al tener la observación de clase "[...] por parte de pares docentes permitirá ver —al menos en parte (pues no es un video sorpresa o cámara escondida) - lo que el docente hace en el salón de clase y la manera de ofrecer conocimiento y motiva el aprendizaje en sus estudiantes [...]" (2016). 
Ciertamente, se evidencia un control y disciplina en esta práctica de autograbación (observación).

Otro rasgo de control y disciplina en las prácticas de observación de clases tiene que ver con las pautas, lineamientos y formatos que debe seguir el observador al realizar el ejercicio. Se controla la práctica, sus resultados y la observación misma al ingresar aciertos o desaciertos del profesor en el formato, en forma de puntos positivos y negativos, si aprobó o no aprobó y hasta comentarios del observador en varios componentes de la clase.

El formato de observación se divide en estructuras precisas sobre lo que debe ver el observador: rendimiento, metas, objetivos, estrategias, materiales, recursos, evaluación y muchas más, para poder controlar y disciplinar las observaciones de clases. Vale decir que hay formatos uniformes-universales que se pueden utilizar para las observaciones, y también algunos de estos son creados por los mismos actores involucrados, ya que conocen sus contextos y dan fe de controlar, mediante un formato, lo que necesita el observador para hacer su trabajo.

Puesto que este artículo tiende a mostrar lo que está más allá de las observaciones de clase, quisiera decir que en referencia a la práctica de autograbación (observación), el profesorado puede decidir si ser o no diferente frente a la cámara. A lo mejor habrá algunos sobreactuados o con circunstancias imprevistas durante las grabaciones, las cuales no se pueden observar o poner en el video. Si bien una buena praxis se hará visible más allá de los pequeños detalles, podrá ocurrir que surjan excelentes actores que graben sus videos y queden catalogados como "buenos(a)" profesores(a) frente a las cámaras.

La observación de clases no siempre detalla el $100 \%$ de la naturalidad de las clases. Según el relato de una profesora a quien se iba a observar: "Profesor M, yo sé que va a observar mi clase mañana y me prepararé para ello [...] estudiaré mi libreto al pie de la letra, seré una gran actriz en el aula de clase, ya lo verá [...]".

Igualmente, los formatos de observación de clases ubican al observador con un panorama limitado; en ocasiones, no hay oportunidad de agregar comentarios extras en este formato, las notas deben ser completadas con el formato que se tiene allí, no hay ocasión de plasmar lo distinto, lo no visible, lo auténtico de las clases del profesorado en estas rúbricas de observaciones de clases. Poco se habla también del momento después de completar ese formato o rúbrica. También es claro que al observador se le escapan situaciones, no todo se escribe, comete equivocaciones, pues finalmente es un ser humano que toma nota de una situación particular en un momento determinado. Por supuesto, el sujeto observador desempeña un papel crucial en este camino del más allá de las observaciones de clases de inglés y es posible argumentar sobre ello.

\section{El sujeto observador}

Llegados a este punto, esta categoría es una de las más analizadas, debido a que no muchos autores han investigado acerca del sujeto observador en las prácticas de observaciones de clases de inglés en Colombia. Se 
considerarán varios elementos que no están descritos y entrarían en la zona del "más allá" de las observaciones de clases de inglés en Colombia.

El observador no es un objeto, es un sujeto. Los observadores tienen sus propias subjetividades, requieren hablar de sus experiencias, de sus deseos, del entorno educativo, de la capacitación como observadores que les ha tocado y de sus intereses. El observador es un hombre que tiene subjetividad y necesita constituirse como sujeto. Este sujeto ha tenido transformaciones de su propia subjetividad, está o ha estado en construcción de un nuevo sujeto. Como lo nombra Quiceno (2004), el sujeto se transforma cuando se encuentra con otras experiencias, en una "modificación del modo de ser de un sujeto" (Foucault, 1994, p.101).

Por tanto, el observador tiene su propia subjetividad o subjetividades. Es importante mencionar que este sujeto se ha venido posicionando en actividades de evaluación, desarrollo profesional y calidad educativa. Muchos de los observadores son llamados "expertos" por sus credenciales y experiencia, tanto académica como disciplinar. El observador de clases ha recibido un entrenamiento para poder realizar una labor pertinente en sus prácticas, debe conocer el contexto en el que se involucra y estar preparado para poder realizar un trabajo de observación eficaz para el caso.

Varios profesores y profesoras que son observados preguntan por este "experto" antes de la observación, ya que requieren ratificar que ese sujeto que se involucrará en su intimidad de prácticas pedagógicas posee gran dominio del idioma (inglés), estudios en alto grado (maestría, estudios en el exterior, exámenes internacionales, entre otros), amplia experiencia como docente y capacitación como observador (varios años en el campo). Todo ello para argumentar validez y posicionamiento cuando se realice la práctica de observaciones. Cuando el profesor observado se da cuenta de que este sujeto tiene todas las características nombradas, ahora sí podrá observar su clase..., "tiene el 'permiso' de ingresar a mi aula para criticar, juzgar, evaluar, realimentar y demás”.

En Colombia pasa frecuentemente que las personas consideran al extranjero como alguien mejor o más capacitado para realizar una labor. En el campo de la enseñanza de inglés no es diferente. Algunas personas consideran que un extranjero observará mejor una clase o hará mejor su labor de observador por el simple hecho de ser extranjero (de un país de habla inglesa, como Estados Unidos, Inglaterra, Australia), sin siquiera tener las credenciales ya citadas. Como ejemplo, en la tabla 1, en la referencia 4, los fellows deberán apoyar a los gestores de bilingüismo en la dinámica de observación de clases en sus visitas a la institución educativa. Con esto, puedo analizar que la posición del sujeto es producida por un programa de gobierno (MEN, 2016) en el que los formadores nativos extranjeros apoyan los procesos educativos al observar las clases del profesorado y validarse, así como sujetos expertos en esta dinámica de observaciones de clases.

El fin de esta categoría es mostrar la variable oculta, la que pocas veces se plasma en los textos o investigaciones sobre observaciones de clases.

Dicho lo anterior, lo que no es visible sobre el sujeto observador se plantea en las negociaciones que hay entre el observador y el observado. 
Esas conversaciones, que no solo nutren la práctica de observación de clases, sino que ayudan a un crecimiento profesional, son un ejercicio de reflexión y mutuo apoyo. Tal como lo dicen Parra y Hernández (2019):

Observar en contexto implica trabajar con una estrategia de observación no estructurada para identificar patrones en eventos de clase y la posterior apertura de espacios para generar diálogos colaborativos (entre observadores y entre observadores y observados), para reflexionar sobre los posibles mecanismos detrás de estos patrones. (p.2)

El profesor no se siente a gusto al ser observado y el observador llega a un acuerdo para hacer de manera diferente esta observación. No hay necesidad de observar, sino que una charla informal, un café antes y/o después de la observación genera más confianza y en gran medida saldrán las variables verdaderas y significativas sobre los procesos pedagógicos de las clases de inglés. Esto es un tanto más humano: dialogar, entender y ponerse en la posición del otro hace que la observación de clases sea un principio ético y humano. ¿Es ello nombrado en los reportes de observaciones de clases?

Los diálogos/narrativas del sujeto observador con y desde el profesorado no salen en los gráficos de mejoramiento de la calidad educativa, no son de gran importancia para triangular información en investigación y muchas veces quedan en el olvido. Creo que estas narrativas entre el observador y el profesorado deben ser sacadas a la luz, porque de allí podrían revelarse posibles cambios y hasta soluciones en estrategias educativas en Colombia.

\section{DISCUSIÓN}

Con el propósito de considerar de manera crítica-reflexiva las prácticas de observaciones de clases de inglés en Colombia (OCIC), es conveniente desarrollar algunas sugerencias, opiniones y otros aspectos respecto al tema.

A continuación, me gustaría hacer visibles algunos detalles de las OCIC. La observación de clases de inglés es un proceso de reflexión en el que las personas involucradas tienen la oportunidad de aprender mutuamente, y va más allá de un control/evaluación de los procesos educativos, tal como lo nombra Lasagabaster (2006):

Es importante que se mantenga el espíritu de observación, es decir, no se pretende supervisar o inspeccionar con una finalidad prescriptiva; no se trata de evaluar al docente, sino de observar qué ocurre en la clase para beneficio de todas las personas inmersas en el proceso de enseñanza-aprendizaje. (p.75)

Las OCIC brindan la posibilidad de trabajar en grupo, de manera colaborativa; en estas, varios sujetos son involucrados en habilidades de escucha, argumentación, análisis, y hacen visible lo no-visible en los contextos escolares, particularmente en las clases de inglés en Colombia. Otro rasgo de las OCIC se sustenta en la capacitación que se debe llevar a cabo para ser observador y encaminar una "buena" observación dentro del aula. Como se ve en la tabla 1 , fuente 5 , una entidad como el British 
Council (Consejo Británico) realiza un paso a paso, seguido de unas recomendaciones, objetivos y aspectos por tener en cuenta mientras se hacen la observación de clases. Si bien es un proceso rígido, como se ha resaltado antes, también hace notar la observación como un proceso estructurado, organizado, fundamentado y validado para obrar de manera eficiente frente a los requerimientos que exige el coordinador, experto, entidad externa, directivos y el gobierno nacional.

Si el lector se para en esta parte de las conclusiones, podrá notar que las observaciones de clases son necesarias como una práctica segura para poder evaluar al profesorado. Sin embargo, la invitación es a dar una mirada sobre ese "más allá" de estas observaciones, analizar las variables que la observación nos brinda y ser un tanto abiertos a eso distinto/ diferente que ofrecen las observaciones dentro del aula de clases.

Como ejemplo, estas son algunas de las razones por las que se ha observado a otro profesor de inglés durante su práctica:

razones por las que se ha observado a otro profesor de inglés durante su práctica

- Para aprender una nueva actividad, método, técnica de clases de inglés.

Para obtener nuevos componentes que ayuden a mejorar en el quehacer pedagógico y humano.

Para llegar a encontrar otros ambientes de aprendizaje y factores diferentes dentro y fuera del aula de clases.

Estas razones no son "recetas" para hacer una observación de clase adecuada; es, más bien, una reflexión en la que se deduce que lo más importante es el acto ético que emerge durante la observación.

En definitiva, las prácticas de observaciones de clases de inglés son, por llamarlas de algún modo, rituales en los cuales están inmersos la gran mayoría del profesorado de inglés, y que, se quiera o no (infortunadamente), deben enfrentarse y vivir con ello. ¿Y la posición de los estudiantes durante estas observaciones?... ¿Acaso no son los estudiantes el eje fundamental en los procesos de enseñanza-aprendizaje? Poco se habló de ellos en este texto, pero seguramente llegar a escribir sobre los estudiantes durante procesos de observación de clases será una experiencia importante y gratificante, así como serán visibilizados en este más allá de las observaciones de clase de inglés en Colombia.

\section{REFERENCIAS}

Aguilar, D., Caruso, M., Echeverri, J., Martínez Boom, A., Narodowski, M., Noguera, C., Quiceno, Zuluaga, O. (2004). Foucault, la pedagogía y la educación. Pensar de otro modo. Editorial Magisterio, Colecciones Pedagogía e Historia.

British Council-Secretaría de Educación Distrital, Convenio 1550. (2017). Guiaspara la Ronda de observación de clase. Escuelas Formadorasy Maestros de Maestros EFMMa. Guía de Rondas de Observación de Clase. 
Cabrales, D. (2019). Las fallas del examen de ascenso docente. Las 2 Orillas. htt ps://www.las2orillas.co/las-fallas-del-examen-de-ascenso-docente/

Foucault, M. (1994). Hermenéutica del sujeto. La Piqueta.

Gosling, D. (2002). Models of Peer Observation of Teaching. Ponencia plenaria en LTSNGC Peer Observation of Teaching Conference. Birmingham. https://www.researchgate.net/profile/David_Gosling/p ublication/267687499_Models_ of_Peer_Observation_of_Teaching/ links/545b64810cf249070a7955d3.pdf

Hernández, P. (2016). ¡Bienvenidos los cambios! Una evaluación integral a los docentes de nuestro país. El Mal Economista. El Espectador. https://blogs. elespectador.com/economia/el-mal-economista/bienvenidos-los-cambio s-una-evaluacion-integral-a-los-docentes-de-nuestro-pais

Hernández, R., Fernández-Collado, C., \& Baptista, P. (2008). Metodología de la investigación. McGraw Hill.

Lasagabaster, D. (2006). La observación de la clase de L2. Revista de Psicodidáctica, 2(11-12). https://www.ehu.eus/ojs/index.php/psicodidac tica/article/view/316

Martínez, A., Cruz, A., Bamond, V., Fernández, I., \& Strotmann, B. (2018). La observación formativa como instrumento de desarrollo en profesores universitarios en la Universidad Europea de Madrid. Revista Complutense de Educación, 29(4), 1365-1380. https://doi.org/10.5209/RCED.55431

Ministerio de Educación Nacional (MEN). (2014). Programa nacional de inglés- Documento de socialización Colombia Very Well 2015-2025. https://www.mineducacion.gov.co/1759/articles343837_P rograma_Nacional_Ingles.pdf

Ministerio de Educación Nacional (MEN). (2016). Colombia BilingüeTeaching Fellowship Program. https://www.mineducacion.gov.co/1621/ articles-132560_recurso_pdf_programa_nacional_bilinguismo.pdf

Ministerio de Educación Nacional (MEN). (2018-2019). Manual para autograbación del video de práctica. Evaluación de Carácter Diagnóstico Formativa (ECDF). https://www.mineducacion.gov.co/1759/articles-3 82455_recurso_3.pdf

Parra, J., \& Hernández, C. (2019). Classroom observation in context: An exploratory study in secondary schools from Northern Colombia. Revista Brasileira de Educação, 24, e240005. Epub. https://dx.doi.org/10.1590/s 1413-24782019240005

Plan de Bilingüismo Municipal Mosquera. (2012).Mosquera Lives English 2012-2021. Plan de bilingüismo municipal "Mosquera Lives English". Recuperado de: https://mosqueracundinamarca.micolombiadigital.gov.c o/sites/mosqueracundinamarca/content/files/000155/7716_mosqueral ivesenglish.pdf

Quiceno, H. (2004). De los usos de Foucault para la práctica pedagógica. Un saber por qué no funciona la escuela. En Aguilar, D., Caruso, M., Echeverri, J., Martínez Boom, A., Narodowski, M., Noguera, C., Quiceno, ...Zuluaga, O., Foucault, la pedagogía y la educación. Pensar de otro modo. Editorial Magisterio, Colecciones Pedagogía e Historia.

Rekalde, I., Vizcarra, M., \& Macazaga, A. (2014). La observación como estrategia de investigación para construir contextos de aprendizaje y fomentar procesos participativos. Educación XX1, 17 (1), 199-220. doi: 10.5944/ educxx1.17.1.1074 
Rodríguez, G., Gil, J., \& García, E. (1996). Metodología de la investigación cualitativa. Málaga: Ediciones Aljibe.

Tinto, J. (2013). El análisis de contenido como herramienta de utilidad para la realización de una investigación descriptiva. Un ejemplo de aplicación práctica utilizado para conocer las investigaciones realizadas sobre la imagen de marca de España y el efecto país de origen. Provincia, (29),135-173. https://www.redalyc.org/articulo.oa?id=555/555304650 07 